\title{
How Classical Particles Emerge From the Quantum World
}

\author{
Dennis Dieks • Andrea Lubberdink
}

Received: 1 February 2010 / Accepted: 22 October 2010 / Published online: 3 November 2010

(C) The Author(s) 2010. This article is published with open access at Springerlink.com

\begin{abstract}
The symmetrization postulates of quantum mechanics (symmetry for bosons, antisymmetry for fermions) are usually taken to entail that quantum particles of the same kind (e.g., electrons) are all in exactly the same state and therefore indistinguishable in the strongest possible sense. These symmetrization postulates possess a general validity that survives the classical limit, and the conclusion seems therefore unavoidable that even classical particles of the same kind must all be in the same state-in clear conflict with what we know about classical particles. In this article we analyze the origin of this paradox. We shall argue that in the classical limit classical particles emerge, as new entities that do not correspond to the "particle indices" defined in quantum mechanics. Put differently, we show that the quantum mechanical symmetrization postulates do not pertain to particles, as we know them from classical physics, but rather to indices that have a merely formal significance. This conclusion raises the question of whether many discussions in the literature about the status of identical quantum particles have not been misguided.
\end{abstract}

Keywords Identical particles · Indistinguishability $\cdot$ Emergence $\cdot$ Classical limit of quantum mechanics

\section{Introduction}

In classical physics, particles are the example par excellence of distinguishable individuals. No two classical particles can be in exactly the same physical state: in Newtonian spacetime different particles will at least occupy different spatial positions at any moment, because of their impenetrability. They will therefore obey Leibniz's

D. Dieks $(\varangle) \cdot$ A. Lubberdink

Institute for the History and Foundations of Science, Utrecht University, P.O. Box 80.010, 3508 TA Utrecht, The Netherlands

e-mail: d.dieks@uu.nl 
Principle of the Identity of Indiscernibles, which says that different individuals cannot share all their physical properties. Moreover, classical particles possess genidentity, i.e. identity over time. That is, given two particle configurations at different instants, it is an objective physical fact for each particle at the later instant with which particle in the earlier configuration it corresponds. This is because classical particles follow definite trajectories that make it possible to follow them over time. Classical particles can thus always be distinguished and be given individual names, or numbers: particle 1, particle 2, etc. These particle numbers are correlated with different and therefore identifying physical characteristics.

In quantum theory the status of individual objects is a notoriously more complicated subject. The standard quantum mechanical treatment of particles starts simply enough, with the uncontroversial case of one particle described in a single Hilbert space. In the case of two or more particles the tensor product of such individual Hilbert spaces is formed, $\mathscr{H}_{1} \otimes \mathscr{H}_{2} \otimes \mathscr{H}_{3} \otimes \cdots$. The natural interpretation, especially with the classical case in mind, is that in such formulas $\mathscr{H}_{i}$ is the Hilbert space of particle $i$ (i.e., the possible states of particle $i$ correspond to density operators defined on $\mathscr{H}_{i}$ ). In other words, it seems natural to interpret the indices as not only referring to the individual factor spaces in the total tensor product Hilbert space, but also to individual particles.

Complications arise for particles of the same kind (so-called "identical particles"). The elementary identical quantum particles we know are either bosons or fermions, whose states-defined in $\mathscr{H}_{1} \otimes \mathscr{H}_{2} \otimes \mathscr{H}_{3} \otimes \cdots \otimes \mathscr{H}_{n}$ - are completely symmetrical or antisymmetrical, respectively. ${ }^{1}$ In such (anti)symmetrical states the restriction of the state to a single factor space (i.e., the density operator obtained by "partial tracing" over the variables of the other factor spaces) is the same for all factor spaces. All one-particle states defined in the individual Hilbert spaces $\mathscr{H}_{i}$ are therefore equal. If the indices $i$ are regarded as particle indices, this means that the several particles cannot be individuated on the basis of their state-dependent properties (like position, momentum, etc.). ${ }^{2}$ Since the state-independent properties (charge, rest mass, etc.) are by definition equal for "identical particles", this leads to the conclusion that all particles of the same kind possess exactly the same physical properties. Their individuality can therefore not be based on individuating physical properties, and Leibniz's Principle (at least the form of it that says that different individuals must differ in at least one of their properties) is apparently violated.

This strange situation is the origin of an extensive literature about the nature of identical quantum particles. The present situation seems to be this: If we do not want

\footnotetext{
${ }^{1}$ We only consider bosons and fermions here, and do not discuss the possibility of paraparticles and parastatistics. However, consideration of paraparticles would not affect the argument of this article.

${ }^{2}$ Here we follow standard interpretational ideas, according to which the states we just mentioned provide complete physical descriptions. If, on the other hand, it is assumed that a finer description is possible, and that the quantum state only provides statistical information about the actual properties of physical systems-like in Bohm's theory or in modal interpretations - there may very well exist individuating physical characteristics. The whole issue is therefore interpretation dependent; our discussion here stays within the standard interpretational framework adopted by most recent discussions about the individuality of identical particles.
} 
the individuality of identical particles to be a fundamental property itself ("haecceity", "fundamental thisness"), there may be the option of weakening Leibniz's principle by introducing a weak form of discernibility, based on the existence of irreflexive relations between the particles (see [2, 5, 15] for general discussion, [9, 10, 12-14] for elaboration of the just-mentioned position, [3, 4] for criticism).

It is important to note that the symmetrization postulates, which are responsible for the equality of all one-particle states, are basic postulates of quantum mechanics that apply to the collection of all particles of the same kind. This means, for example, that all electrons in the universe are in exactly the same state, whatever the differences between the physical conditions at different positions in the universe. In particular, it does not make sense to distinguish between electrons here and electrons elsewhere, for instance in another solar system: all electrons in the universe are localized in exactly the same way ("spread out over all electron positions", see below for more on this). It is not relevant for this universal applicability of the symmetry postulates what kinds of interactions and situations are considered; consequently, whatever circumstances may turn out to be important for the transition to the classical limit, these do not affect the applicability of the symmetrization postulates. This implies that even in the classical limit the different particle indices $i$ are all associated with exactly the same state. In other words, it seems that even classical particles must be completely indistinguishable!

This result is obviously problematic - in fact, we began our whole argument by pointing out that classical particles are distinguishable objects par excellence. So something must have gone wrong in the above reasoning. In the remainder of this article we shall analyze the source of this paradox, and investigate the implications of our analysis for the particle concept in quantum mechanics.

\section{The States of Identical Particles}

Consider the concrete case of a system consisting of two electrons. ${ }^{3}$ Electrons are fermions and therefore have an antisymmetrical state, typically looking like

$$
|\Psi\rangle=\frac{1}{\sqrt{2}}\left(|\phi\rangle_{1}|\psi\rangle_{2}-|\psi\rangle_{1}|\phi\rangle_{2}\right) .
$$

Here, the subscripts 1 and 2 indicate whether the indexed state is defined in $\mathscr{H}_{1}$ or in $\mathscr{H}_{2}$, respectively. Taking partial traces, we find that both the state restricted to $\mathscr{H}_{1}$ and the state restricted to $\mathscr{H}_{2}$ has the form $W=\frac{1}{2}(|\phi\rangle\langle\phi|+| \psi\rangle\langle\psi|)$. If we think of $\mathscr{H}_{1}$ and $\mathscr{H}_{2}$ as the state space of particle 1 and particle 2 , respectively, we can thus conclude that both particles are in exactly the same state: figuratively speaking, they are both half in $|\phi\rangle$ and half in $|\psi\rangle$.

\footnotetext{
${ }^{3}$ As just explained, we should in principle always consider the fully entangled state of all electrons in the universe and, in view of the equality of all partial traces, considering a two-electrons subsystem with specific properties does not really makes sense then. So at the moment it is best to think of a universe in which there exist only two electrons. In Sects. 3 and 4 we shall work out a particle concept for which it does make sense to consider specific subsystems.
} 
This means that it would not be correct to say that $|\Psi\rangle$ describes one particle in $|\phi\rangle$ and one in $|\psi\rangle$. In fact, a state with particle 1 in $|\phi\rangle$ and particle 2 in $|\psi\rangle$ necessarily would have to possess the product form $|\phi\rangle_{1} \otimes|\psi\rangle_{2}$ [16, sect. VI.2], which not only conflicts with the symmetrization postulate but is also empirically different from (1). The expectation value of an observable $A$ of the two-electron system (a symmetrical hermitean operator ${ }^{4}$ in state (1), $\langle\Psi|A| \Psi\rangle$, differs from its expectation value in a product state by the presence of an interference term $\left\langle\phi_{1} \otimes \psi_{2}|A| \psi_{1} \otimes \phi_{2}\right\rangle$. It may happen, of course, that this cross term vanishes for particular choices of $A$, and in this case use of the product state does not lead us into conflict with empirical results. But then there always are other observables for which the cross terms do not vanish, and empirical evidence confirms the existence of these terms. This means that the suspension of the symmetrization postulates that sometimes occurs in the physics literature, for instance when spatially isolated systems are subjected to position measurements, is only pragmatically justified. This manoeuvre simplifies the calculations, but has no fundamental status. The fully symmetrized entangled state has general applicability and validity, and is therefore the only one to be used in a general analysis.

The conclusion is thus unavoidable that different "particle indices" $i, j$ cannot be associated with any measurable physical differences. Among philosophers of physics this is an acknowledged fact that has given rise to the hotly debated question of what then $i$ able to ground the individuality of these particles.

But it should be noted that in those parts of the foundational literature that do not focus on identity issues, and in the actual practice of physics, the use of the particle concept is not unequivocal. It is true that particles are sometimes associated with the indices of our above discussion; but one also encounters another, very different use of the particle concept (cf. $[6,7]$, where a distinction is introduced between "h particles" and "q particles"). This alternative approach is significant for our later analysis, and we therefore want to illustrate it by an example, namely the notorious EinsteinPodolsky-Rosen case.

In its modern version, the EPR experiment refers to two electrons "at a distance from each other", on which spin measurements are performed. The two-particle spin state on which attention usually focuses is the singlet state, but the full state obviously also contains a spatial part. A correctly symmetrized total state is

$$
|\Phi\rangle=\frac{1}{\sqrt{2}}\left(|\phi\rangle_{1}|\psi\rangle_{2}+|\psi\rangle_{1}|\phi\rangle_{2}\right)\left(|\uparrow\rangle_{1}|\downarrow\rangle_{2}-|\downarrow\rangle_{1}|\uparrow\rangle_{2}\right),
$$

where $|\uparrow\rangle$ and $|\downarrow\rangle$ stand for spin eigenstates with spin directed upwards and downwards in a particular direction, respectively, and where $(|\phi\rangle$ and $|\psi\rangle$ now refer to states that are localized "on the left hand side" and "the right hand side", respectively. ${ }^{5}$ In the language of wave mechanics, $|\phi\rangle$ and $|\psi\rangle$ represent localized wave packets at a macroscopic distance from each other.

\footnotetext{
${ }^{4}$ All observables of an identical particle system have to be symmetrical, among other things in order to preserve the symmetry properties of the states over time. This has to be imposed in addition to and independently of the (anti)symmetrization of the states.

${ }^{5}$ In the state 2 there is no correlation between positions and spins. An alternative state, in which there is such a correlation, is $|\Phi\rangle=\frac{1}{\sqrt{2}}\left(|\phi\rangle_{1}|\psi\rangle_{2}|\uparrow\rangle_{1}|\downarrow\rangle_{2}-|\psi\rangle_{1}|\phi\rangle_{2}|\downarrow\rangle_{1}|\uparrow\rangle_{2}\right)$. Consideration of this alternative
} 
In the literature on EPR the state is often given in a different form, namely

$$
\left|\Phi^{\prime}\right\rangle=\frac{1}{\sqrt{2}}|\phi\rangle_{1}|\psi\rangle_{2}\left(|\uparrow\rangle_{1}|\downarrow\rangle_{2}-|\downarrow\rangle_{1}|\uparrow\rangle_{2}\right),
$$

in which the spatial part is a simple product state. Clearly, this state does not obey the anti-symmetrization postulate, and from a fundamental point of view it therefore cannot be right. It is true that as long as we only consider observables that commute with position, we shall not arrive at any wrong empirical predictions, and this yields a pragmatic justification for using (3). But the really important advantage of using (3) instead of the correct state is that this form of the state lends itself to an easy interpretation: we have one particle at the left hand side, and one on the right hand side. This fits in with the standard way of speaking about EPR. According to the usual discussions there is a left-side particle $L$ and a right-side particle $R$, and we are interested in the results of spin measurements on these two individual particles. Note that in this common way of dealing with the situation the particles are treated as individuals that differ from each other in their physical properties, namely their locations.

But if we use the correct form (2), and associate our particles, in accordance with the official doctrine, with the "particle indices" 1 and 2, we have to conclude that there is no left and no right particle: the states of both 1 and 2 are "evenly distributed" between left and right. This means that the way the EPR case is usually understood, as being about a particle $L$ and a particle $R$, is at variance with the official doctrine regarding the concept of particles in quantum mechanics.

Of course, those who think in terms of individual localized particles in this case (i.e., in practice almost everyone) generally know that the state in principle has a form like (2); but this does not induce them to abandon the idea of an individual $L$ and an individual $R$ particle. This points into the direction of the existence of an alternative way of handling the particle concept, one that does not relate particles to the indices in the tensor product formalism. Apparently, such an alternative conception is already present in the practice of physics - at least on an intuitive level. As we shall see, if worked out this other way of interpreting the particle concept, rather than the official doctrine that indices represent particles, provides a natural bridge to particles as they occur in classical physics.

\section{Classical Particles in Quantum Mechanics}

Classical particles are characterized by their unique spatial positions and trajectories. It is often said that both these features are excluded in quantum mechanics; and that therefore in quantum mechanics it cannot be an objective fact which particle at a later instant is identical with which earlier particle. According to this argument the concept of genidentity does not apply to quantum particles. However, if this absence of particle localization and particle trajectories were a matter of principle, the resulting situation would be very puzzling. Surely, the classical particle picture must be

state leads to the same conclusion as the discussion in the main text: the indices 1,2 do not refer to localized particles. 
expected to emerge from quantum mechanics in some limiting case, and one must therefore assume that the typical classical particle features can be mimicked in quantum mechanics. In fact, that this is indeed the case is well known, in spite of the declarations to the contrary that we have just mentioned. One key result in this connection is Ehrenfest's theorem about the dynamics of expectation values of observables.

In the case of a Hamiltonian $H=p^{2} / 2 m+V(r)$, with $p$ the momentum, $m$ the particle mass and $V(r)$ a potential field, we can introduce a force field $F(r)=$ $-\nabla V(r)$, in terms of which Ehrenfest's theorem takes the form

$$
\langle F(r)\rangle=m \frac{d^{2}}{d t^{2}}\langle r\rangle .
$$

For certain specific potentials (free motion, i.e. $F=0$, or if $V$ is a quadratic function of $r$ ) we find that $\langle F(r)\rangle$ equals $F(\langle r\rangle)$, so that in these cases the mean value of $r$ exactly satisfies the classical law of motion $F(\langle r\rangle)=m \frac{d^{2}}{d t^{2}}\langle r\rangle$. In general this is not so. But if the wave function is localized in a sufficiently small region of space, so that the variation of the force field within that region is small, we can replace (4) by the classical equation in a good approximation (which becomes better when the state becomes more localized). From this it follows that well-localized single-particle quantum states (localized in the sense that their associated wave packets are very narrow) follow classical trajectories to a very good approximation.

Classical trajectories therefore do exist in quantum mechanics: they are realized by (very) small wave packets. In the case of a Hamiltonian that is quadratic in positionthe harmonic oscillator being the prime example—such small wave packets remain small over time: their widths merely oscillate. This case therefore furnishes an example of a quantum system that almost perfectly mimics the behavior of a classical particle.

If the potential does not have this special form there will in general be dispersion, so wave packets will spread out. Classical motion will then only be a good description of the behavior of the average position of the wave packet during a finite time, during which the approximation $\langle F(r)\rangle=F(\langle r\rangle)$ remains valid. Moreover, even if the center of the wave packet stays on a classical trajectory, the analogy with a classical particle path will get partially lost if the packet becomes too extended. Free motion furnishes one example: although in this case the average position of a moving wave packet will always be exactly on the classical trajectory, the width of the packet will increase in an approximately linear way, according to $\Delta r=\left\{\left(\triangle r_{0}\right)^{2}+\left(\triangle p_{0} t / m\right)^{2}\right\}^{1 / 2}$ (with $t$ representing time). When the size of the packet has become substantial, results of consecutive position measurements will no longer need to lie on a classical path, not even approximately. A classical particle picture then does not apply. Consequently, we need a mechanism to keep wave packets narrow in order to maintain classical particle-like structures in quantum mechanics over longer stretches of time.

Such considerations are standard in studies on the classical limit of quantum mechanics, and there is growing agreement that the essential element in explaining how classical mechanics emerges from quantum mechanics is the process of decoherence. The key ideas are that physical systems are usually not isolated but interact with an environment; and that in many circumstances the interaction is such that the environment effectively performs (approximate) position measurements on the systems in 
question. The effect of this interaction with the environment is the destruction of coherence between parts of the wavefunction centered around different positions: these parts become correlated with mutually orthogonal environment states. Spatially extended wave functions are transformed into mixtures of spatially very narrow states. Model calculations indicate that these narrow wave functions obey the quantum mechanical evolution equation governed by the system's own Hamiltonian (leading, among other things, to the validity of Ehrenfest's theorem commented upon above) plus two terms representing the interaction with the environment (see [17], especially equations 17 and 24 therein). The first of these terms is a damping term, representing friction with the environment; the second term, more important for our purposes, represents the decoherence process and keeps on minimizing the dimensions of the wave packet.

As a result, the classical limit of quantum mechanics is characterized by the emergence of classical particle trajectories that are followed by narrow wave packets. These narrow, localized wave packets become the particles we are familiar with in classical physics. Collections of such localized wave packets represent the particle subsystems we are used to refer to (compare footnote 2 in Sect. 2).

\section{The Particle Concept in Quantum Mechanics}

The finer details of the decoherence mechanism, and the work that remains to be done to fully understand them, need not detain us here. The important thing is that there is a consensus that classical particles emerge from quantum mechanics as narrow wave packets that in very good approximation follow classical particle paths. This is the conceptual background of what we have signalled before: in the practice of physics the particle concept is very often not linked to the indices in the formalism, but rather to distinct localized states. The EPR experiment, where the localized states on the left and right wing of the experiment are associated with an individual $L$ and $R$ particle, respectively, is but one example of this. The way we usually speak about experiments (the positrons in the CERN experiment, etc.) or about the objects surrounding us (the quantum particles making up this table) are other examples.

Thinking about quantum particles in this way is eminently reasonable. The origin of the concept "particle" comes from classical physics, and in this classical context we know exactly what we are talking about when we use the term. Our language is permeated by concepts referring to localized objects and particles. Given this background, it seems only natural to reserve the same term in quantum mechanics for things that share core characteristics with classical particles and that become recognizable as classical particles in the classical limit. This bill is fitted by localized states, but not by the states associated with "particle indices".

To see the difference between the two rival quantum particle concepts clearly, consider the state

$$
\frac{1}{\sqrt{2}}\left(|\phi\rangle_{1}|\psi\rangle_{2}+|\psi\rangle_{1}|\phi\rangle_{2}\right)
$$

in which the one-particle states $|\phi\rangle$ and $|\psi\rangle$ are localized. It could be that they have always been this way, and that the dynamics preserves the localization (as in the harmonic oscillator case), but the more typical situation is that decoherence processes 
are responsible for the appearance of this localized two-particle state in a incoherent mixture of similar states. Now, according to the way of handling the particle concept that we have just explained, the state (5) represents two individual and distinguishable particles, one at the position where $|\phi\rangle$ is localized and the other at the position defined by $|\psi\rangle$. By contrast, if we hold fast to the idea that particles are represented by the indices occurring in the formalism, we arrive at the conclusion that (5) represents a situation in which there are two indistinguishable "particles", both in the state $\frac{1}{2}(|\phi\rangle\langle\phi|+| \psi\rangle\langle\psi|)$. As we have mentioned before, this indistinguishability survives the classical limit: since all factor Hilbert spaces and the states defined in them occur completely symmetrically in the total state, all interactions will affect the states in the factor spaces in exactly the same way. So all indices will remain associated with the same density operator, evenly distributed over the pure one-particle states. The "index-particles" therefore do not become classical particles in this limit: they refuse to become localized. This seems a reductio of the idea that the Hilbert space indices can be taken to stand for particles.

\section{Emergence of Particles in Quantum Mechanics}

Our proposal is therefore to think of particles in quantum mechanics as represented by localized wave packets. That is to say, if we encounter a state $|\Psi\rangle$ defined in $\mathscr{H}_{1} \otimes \mathscr{H}_{2} \otimes \mathscr{H}_{3} \otimes \cdots \otimes \mathscr{H}_{n}$, and wish to investigate whether it can be interpreted in terms of particles, we have to ask ourselves whether it can be written as a symmetrized product of localized one-particle states. A worry that might arise here is whether such a decomposition of $|\Psi\rangle$, if it exists, is unique. If more than one particlelike representations of $|\Psi\rangle$ could be found, the uniqueness of the classical limit and the meaningfulness itself of our particle concept would be endangered. At first sight this worry seems certainly serious, because the symmetrization postulates require that the coefficients appearing in front of the product terms in the symmetrized state $|\Psi\rangle$ are all equal. For example, in state (5) both terms are prefixed by $\frac{1}{\sqrt{2}}$, which means that we are dealing with a degenerate Schmidt (bi-orthogonal) decomposition. In such a case there are infinitely many other Schmidt decompositions: each rotation in the subspaces of $\mathscr{H}_{1}$ and $\mathscr{H}_{2}$ spanned by $|\phi\rangle$ and $|\psi\rangle$ leads to a new pair of vectors $\left|\phi^{\prime}\right\rangle,\left|\psi^{\prime}\right\rangle$ in terms of which the bi-orthogonal form (5) can be written down too. However, and this is crucial, these alternative decompositions will not be in terms of localized wave packets. Indeed, rotations in Hilbert space are implemented by unitary transformations that transform the original vectors into linear combinations of them; if the original states are localized in connected regions of space the transformed stated, being superpositions of the original ones, are obviously not thus localized. It follows that if a decomposition of state $|\Psi\rangle$ is possible in form (5) with localized states $|\phi\rangle$ and $|\psi\rangle$, this decomposition is unique. This argument generalizes immediately to the case of an arbitrary number of particles.

The demand that a state represents particles, in the sense we have defined here, is therefore much stronger than that the state can be written in the form (5) with mutually orthogonal $|\phi\rangle$ and $|\psi\rangle$. The latter is always possible, for any state in a Hilbert space $\mathscr{H}_{1} \otimes \mathscr{H}_{2}$ (because of Schmidt's theorem). It is the added localizability 
condition that makes the question of whether there exists a decomposition of the required form non-trivial, and makes the decomposition unique if it exists.

In most cases states will not allow a particle interpretation; think, for example, of a state of the form (5) with two overlapping wave packets $|\phi\rangle$ and $|\psi\rangle$ (each defined in a connected region of space). The bi-orthogonal decomposition that we need, in terms of localized states that are non-overlapping (and therefore mutually orthogonal) clearly does not exist: there of course does exist a bi-orthogonal decomposition, but the states occurring in it will be linear combinations of $|\phi\rangle$ and $|\psi\rangle$ and will therefore overlap spatially. An arbitrarily chosen quantum state will therefore not describe particles. We need special circumstances to make the particle concept applicable. In this sense, the classical limit with its decoherence processes makes classical particles really emerge from the substrate of the quantum world.

It should be added that the circumstances that are responsible for the emergence of classical particles also justify the use of the statistics that we expect for the case of several independent individuals. The symmetrization postulates require that "manyparticle states" (in the sense of general states in a Hilbert space that is the tensor product of more than one factor spaces) are entangled, and in general this leads to the existence of correlations in measurement results, even if there is no question of past or present mutual interactions. From our perspective, this remarkable "quantum statistics" (either Fermi-Dirac or Bose-Einstein) points into the direction of a failure of the individual particle concept in the general quantum situation. The "particle alternative" is to see the existence of these correlations as a sign that quantum particle states are subject to peculiar initial or boundary conditions (see $[2,5,15]$ for discussion), or that quantum particles exert "exchange forces" on each other (repulsion between fermions and attraction between bosons, see [8] for a critical discussion of this concept). In our approach complications of this kind do not arise, since we reject the idea of particles in the general situation in which we do not have localized systems. In the case of spatially non-overlapping wave packets, in which the particles concept does become applicable, both Fermi-Dirac and Bose-Einstein statistics reduce to the usual Boltzmannian statistics, as is well known.

\section{Classical Particles and Indices}

In order to obtain a clearer view on the connection between particles as we have defined them here, via localized wave packets, and the particle concept that relates to the indices in the formalism, it may be helpful to compare with an analogy that may be constructed in classical mechanics [6,7]. As it turns out, it is possible to define classical "indistinguishable particles" that resemble the indistinguishable "index-particles" in quantum mechanics. ${ }^{6}$

We do not ordinarily use symmetry postulates in classical mechanics, but in the case of particles of the same kind we could introduce a symmetrization procedure

\footnotetext{
${ }^{6}$ The formal structure of quantum mechanics is essentially different from that of classical mechanics, in particular because superpositions make no sense classically. The analogy presented here can therefore only be partial.
} 
without changing the empirical content of the theory. In the usual formalism the state of a system consisting of $n$ particles of the same kind is represented by one point in phase space, with the first coordinate standing for the position of particle 1 , the second for the position of particle 2 , the $(n+1)$-th coordinate representing the momentum of particle 1, etc. Obviously, it would not make any empirical difference if we were to call particle 1 particle 2 , etc. The only thing that is important is that there is one particle in state $\left(x_{1}, p_{1}\right)$, one in $\left(x_{2} . p_{2}\right)$, etc.; the states individuate the particles and it is irrelevant how we number them. Permutation of the names of the particles will not lead to any physical differences. With this in mind, consider all permutations of the particle numbers, in which these are distributed differently over the one-particle states. This will generate $n$ ! phase points, in which the individual oneparticle states are numbered differently, corresponding to the number (name) of the particle to which they pertain. These $n$ ! states are all empirically indistinguishable from each other and from the original state, the only difference between them being the way the one-particle states are indexed, i.e. on which phase space axes each particular one-particle state $(x, p)$ is represented. Now, instead of the usual mechanical state, given by our single original phase point, we might introduce a symmetrized state represented by the complete collection of these $n$ ! points. This new state is symmetrical because it is invariant under permutations of the indices. All the usual formulas from classical mechanics can be reformulated to accommodate this new state definition: the idea simply is to do the usual calculations for each point separately. For the case of dynamical evolution this will lead to a new $n$ !-points state, again symmetrical and with all points empirically equivalent; and in general, the calculations will lead to $n$ ! results that only differ from each other in their assignments of indices. The final result can then be taken as the collection of these $n$ ! partial results.

Evidently, the sole purpose of this manoeuver is to make it manifest that nothing physical depends on the numbering of the particles. The particles are physically characterized by the individual states $(x, p)$, not by the indices. But now suppose that, in spite of this symmetrization, we are caught in the idea that each index has to correspond to a specific particle, and that we are going to inquire about the state of particle $i$. In our symmetrized scheme, the natural answer consists in the collection of $(x, p)$ states that bear the index $i$, given the $n$ ! phase points that make up the many-particle state. In this way all one-particle states are attributed to each index value. The conclusion would then be that the particles are all in exactly the same state and therefore indistinguishable.

It goes without saying that the latter conclusion is in conflict with the way the particle concept is actually used in classical mechanics. In classical mechanics particles are as distinguishable as their states $(x, p)$ are; and from this point of view the above argument is simply a confused misinterpretation of the indices. The indices were only formal expedients, but the argument took them to denote individual physical objects. The resulting indistinguishability paradox is dispelled once we realize what role the indices really play.

This mistaken piece of classical arguing is, however, analogous to the standard reasoning we find in quantum mechanics: instead of looking for individuating physical particle characteristics that might make it possible to speak of individual, distinguishable entities, one holds fast to the a priori idea that the Hilbert space indices should 
play this role. The symmetry of the formalism should give one pause: instead of indicating that all particles are in the same state, this symmetry signals that the indices do not have the role of particle names.

\section{Weak Discernibility}

In the foregoing sections we have focused on the classical limit of quantum mechanics, with the aim of showing that the indices in the tensor product formalism do not become classical particle names in this limit. This leaves it open, however, that the indices refer to individual objects of a different kind, genuine quantum particles, say, that remain distinct from the classical particles that emerge in the classical limit. There is indeed a growing literature in which it is claimed that the indices in the tensor product formalism do refer to such individual physical entities, distinct from each other by virtue of their physical characteristics. This is clearly a claim that has to be investigated. Associating each index with its own haecceity in order to guarantee that it corresponds to an individual object is a move that needs not be taken very seriously, as it boils down to attributing individuality by fiat; but if it is true that there are physical features that discern the indices, the conclusion that they are denoting individual quantum objects becomes harder to resist.

We have already seen that all indices are associated with exactly the same reduced state; this seems to make the existence of individuating physical properties impossible from the outset. However, there is a way out on which the approach we just alluded to is based. The core idea is in the observation that even within the scope of classical physics situations are thinkable in which entities are in identical states but are nevertheless distinct individuals, namely situations with complete symmetry. A famous example was introduced by Max Black [1]: consider two spheres of exactly the same form and material constitution, alone in a relational space (in order to exclude absolute position as a distinguishing property), at a fixed distance from each other. This is a situation that seems certainly thinkable without getting into contradictions. But it is also a situation in which no physical features are able to distinguish between the two spheres, in spite of the fact that there are obviously two of them. The spheres thus seem to defy Leibniz's Principle, and appear to possess an identity that cannot be grounded in physical differences.

However, there is a way to save a form of Leibniz's Principle in such symmetrical classical configurations. As pointed out by Saunders [12, 13], who takes his cue from Quine [11], irreflexive relations are instantiated here: relations entities cannot bear to themselves. In the case of the spheres, each sphere has a non-vanishing distance to one other sphere; and an object cannot possess such a distance to itself. This irreflexivity is the key to proving that (a generalized version of) Leibniz's Principle is satisfied after all. If an entity stands in a relation that it cannot have to itself, there must be at least two entities. It is not difficult to formalize this argument and to prove that the existence of irreflexive physical relations grounds the multiplicity of objects, without recourse to haecceities. Because of the sameness of all individual states it still is impossible to give names based on physical characteristics in such cases; for example, we cannot give a description of one of our spheres that would not apply 
equally to the other one. The objects are therefore not distinguishable in the usual sense; but still we can prove that there are more than one of them. For this reason the term "weak discernibility" has been introduced to capture how objects differ from each other in such situations.

The idea now is that in quantum mechanics the situation is analogous. That is, although the states associated with different indices are identical, irreflexive relations exist between the indices that make them weakly discernible. In the case of fermions the total state is antisymmetric, like in (1), and here the irreflexive relation takes the form of "being associated with different one-particle states". Indeed, as can be verified in (1), in the antisymmetric case each term of the superposition contains indices that indicate different vectors in Hilbert space. From this Saunders $[9,12,13]$ concludes that fermions are physical individuals, albeit only weakly discernible ones. Muller and Seevinck extend this argument to bosons [10]. They observe that quite generally there exist irreflexive relations between the indices: operators that belong to different Hilbert spaces, indexed by different indices, always commute, whereas this is not the case for operators belonging to the same Hilbert space. In particular, momentum and position operators with different indices commute, whereas they do not if their indices are the same. So even bosons appear to be weakly discernible individuals.

It should be noted, however, that these arguments hinge on a silent premiss, namely that the indices not only play a mathematical role but also possess physical significance. As mathematical demonstrations, demonstrating the individuality of the different factor spaces, they are unproblematic; but we need an additional justification for thinking that the indices also correlate to something physical. Of course, it is simple enough to find irreflexive relations between numbers, for instance the relation of inequality. It is also easy to couch such relations in a language that suggests reference to physical quantities, for instance by speaking about observables that belong to the same or different Hilbert spaces. But that will not suffice. We need a positive indication that the different Hilbert space, and the operators ("observables") defined in them, refer to elements of the physical world - certainly not all mathematical quantities occurring within a physical theory refer to things existing physically. In our case, whether the Hilbert space indices and the mathematical quantities labelled by them possess physical significance is precisely the issue under discussion, and it would therefore be question begging to assume this significance. We need an argument to make the indices physically respectable. To get a clue about possible criteria here, let us first have a look at classical physics.

In situations in classical physics without particular symmetries, physical relations can be used to distinguish and name the things that are related. For example, in an arbitrary configuration of more than two classical particles the distances with respect to the other particles will unambiguously characterize each individual particle, and in this way we obtain clear evidence that the relata in distance relations are separate physical objects. In other words, distance relations are the kind of relations that connect physical things. Changing the configuration so that it becomes more symmetrical (but not yet completely symmetrical) will change the values of the distances, but not the number or nature of the objects themselves. This possibility of distinguishing and naming actual objects in asymmetrical situations thus provides us with a justification 
for thinking that if distance relations apply, it is physical things to which they apply. The completely symmetrical situation is a degenerate situation, a limiting case, in which naming via distances admittedly becomes impossible but in which the distance relations are still sufficient to establish weak discernibility and are able to fix the number of objects.

Indeed, why are we so sure intuitively that there are two Blackean spheres? This is because our mind's eye sees these spheres at different distances or in different directions before us; in thought we break the symmetry, which makes it possible to distinguish the entities and name them (the left and right sphere, for example). The symmetrical configuration is thus thought of as a limiting case of the more familiar asymmetrical situation.

Now compare the quantum case. Can a similar story be told here, to make it acceptable that the indices are potential particle labels? Unfortunately, this attempt is immediately thwarted by the symmetrization postulates. It is a fundamental principle of quantum theory that the indices can never appear in configurations that are not symmetrical. In classical physics perfect symmetry of particle configurations, if it occurs at all, is something contingent; but in quantum mechanics it is a law-like feature that all indices must always occur, in any expression and in whatever situation, in a fully symmetrical way. It is even useless to introduce an external standard: if in thought we inject ourselves into the world of electrons, quantum theory requires that all relations between us and the electrons remain completely symmetrical in the indices. This is very much different from the case of Black's spheres. In quantum mechanics it is a matter of principle that we can never associate different physical characteristics with different indices in the formalism. We therefore lack evidence that the indices may refer to distinct physical entities at all (see for more on this $[3,4]$ ), and the irreflexive relations in which the indices stand can not be assumed to connect physical entities.

But what about our actual experience, telling us that in many experiments we do encounter individual electrons and other particles? This we have discussed in the previous sections: such experiments, to the extent that they provide convincing evidence about the presence of particles, pertain to classical limiting situations. As we have seen, the particles that emerge in those situations do not correspond to the indices in the quantum formalism.

\section{Conclusion}

We conclude that the indices in the quantum mechanical formalism of "identical particles" refer to the individual factor spaces from which the total Hilbert space in the formalism is constructed - they are merely mathematical quantities.

In order to support this conclusion we have first argued that, within a standard no-hidden-variables interpretational context, the classical limit does not associate Hilbert space indices with particles as we know them from pre-quantum physics. Well-localized wave packets do take on this role: they do represent classical particles in the limiting situation. The appearance of particles in quantum mechanics is therefore a case of emergence. Only if specific physical conditions are satisfied, resulting 
in the presence of localized wave packets (decoherence processes are usually essential here) does the concept of a particle in the ordinary sense become applicable to the world described by quantum mechanics. These emerging particles are not linked to the indices occurring in the formalism.

Second, we have argued more generally that in the standard interpretation there is no indication that the indices in the formalism denote distinct physical entities at all. Rather, the symmetrization postulates have the effect of eliminating any potential physical label-like role of the indices. The analogy between quantum mechanical systems of "identical particles" and classical collections of symmetrically positioned weakly discernible objects is only superficial. There is no sign within standard quantum mechanics that "identical particles", denoted by indices, are physical objects at all.

This conclusion raises the question of whether the discussions in the philosophy of physics about the nature of the individuality of identical quantum particles have not been misguided. It makes little sense to wonder whether Leibniz's Principle is satisfied by identical quantum particles, or whether they possess haecceities, if the existence itself of these particles has not been established.

Open Access This article is distributed under the terms of the Creative Commons Attribution Noncommercial License which permits any noncommercial use, distribution, and reproduction in any medium, provided the original author(s) and source are credited.

\section{References}

1. Black, M.: The identity of indiscernibles. Mind 61, 153-164 (1952)

2. Dieks, D.: Quantum statistics, identical particles and correlations. Synthese 82, 127-155 (1990)

3. Dieks, D., Versteegh, M.A.M.: Identical particles and weak discernibility. Found. Phys. 38, 923-934 (2008)

4. Dieks, D.: Are 'identical quantum particles' weakly discernible objects. In: Suarez, M., Dorato, M., Redei, M. (eds.) EPSA Philosophical Issues in the Sciences: Launch of the European Philosophy of Science Association, vol. 2, pp. 21-31. Springer, Berlin (2010)

5. French, S., Krause, D.: Identity in Physics: A Historical, Philosophical, and Formal Analysis. Oxford University Press, London (2006)

6. Lubberdink, A.: De individualiseerbaarheid van identieke deeltjes. Master Thesis, Utrecht University (1998). http://gradthesis.andrealubberdink.nl

7. Lubberdink, A.: Identical particles in quantum mechanics. arXiv:0910.4642

8. Mullin, W.J., Blaylock, G.: Quantum statistics: is there an effective fermion repulsion or boson attraction? Am. J. Phys. 71, 1223-1231 (2003)

9. Muller, F.A., Saunders, S.: Discerning fermions. Br. J. Philos. Sci. 59, 499-548 (2008)

10. Muller, F.A., Seevinck, M.: Discerning elementary particles. Philos. Sci. 76, 179-200 (2009)

11. Quine, W.V.: Grades of discriminability. J. Philos. 73, 113-116 (1976). Reprinted in Quine, W.V.: Theories and Things. Harvard University Press (1981)

12. Saunders, S.: Physics and Leibniz's principles. In: Brading, K., Castellani, E. (eds.) Symmetries in Physics: Philosophical Reflections, pp. 289-307. Cambridge University Press, Cambridge (2003)

13. Saunders, S.: Are quantum particles objects? Analysis 66, 52-63 (2006)

14. Teller, P.: Quantum mechanics and haecceities. In: Castellani, E. (ed.) Interpreting Bodies: Classical and Quantum Objects in Modern Physics, pp. 114-141. Princeton University Press, Princeton (1998)

15. van Fraassen, B.: Quantum Mechanics: An Empiricist View. Oxford University Press, London (1991)

16. von Neumann, J.: Mathematische Grundlagen der Quantenmechanik. Springer, Berlin (1932) and (1996)

17. Zurek, W.H.: Decoherence and the transition from quantum to classical-revisited. In: Duplantier, B., Raimond, J.-M., Rivasseau, M. (eds.) Quantum Decoherence, Poincaré Seminar 2005. Progress in Mathematical Physics, vol. 48, pp. 1-31. Birkhäuser, Basel (2007) 